\title{
Impacto do Confinamento em Doentes com Esquizofrenia Durante a Pandemia de COVID-19
}

\section{Consequences of the Lockdown in Patients with Schizophrenia During the COVID-19 Pandemic}

Joana Lopes ${ }^{1 *}$, Inês Silva², Vera Reynaud da Silva³

\section{RESUMO}

INTRODUÇÃO: A pandemia de COVID-19 e as medidas de saúde pública implementadas para a controlar podem causar ou acentuar sintomas de ansiedade e depressão, especialmente em pessoas vulneráveis, como os doentes com esquizofrenia.

MÉTODOS: O presente estudo procurou avaliar o impacto do confinamento de março e abril de 2020 numa amostra de 40 doentes com esquizofrenia acompanhados na consulta externa do Hospital de Vila Franca de Xira.

RESULTADOS: Destes doentes, 65\% respeitaram o confinamento, 32,5\% sentiram maior ansiedade, 97,5\% mantiveram a medicação e 15\% aumentaram as suas doses. Os consumos de álcool aumentaram em 42,8\% dos doentes e os de tabaco em 30,7\%, mas não houve aumento nos consumos de cannabis. Houve um aumento na procura de ajuda psiquiátrica em 10\% dos doentes e psicológica em 15\% dos casos.

CONCLUSÃO: Não houve redução da adesão à terapêutica e a maioria dos doentes cumpriu as regras de confinamento impostas.

PALAVRAS-CHAVE: COVID-19; Esquizofrenia; Pandemia 


\section{ABSTRACT}

INTRODUCTION: COVID-19 pandemic and the public health measures adopted to restrain its spread can cause or accentuate symptoms of depression and anxiety, especially in vulnerable populations, like schizophrenia patients.

METHODS: The current study evaluated the impact of the lockdown measures applied in March and April 2020 in Portugal, in a group of 40 ambulatory patients with schizophrenia from Hospital Vila Franca de Xira.

RESULTS: In this group of patients, 65\% respected the lockdown measures, 32.5\% had higher anxiety levels, $97.5 \%$ maintained their medication and $15 \%$ raised the dosage. Alcohol doses were higher in $42.8 \%$ of regular drinkers and tobacco doses were higher in $30.7 \%$ of smokers but there was no raise in cannabis use. A percentage of $10 \%$ of patients had urgent psychiatric consultations and 15\% of patients felt the need for psychological support.

CONCLUSION: There was no significant loss of adhesion to medication and most patients complied with lockdown measures.

KEYWORDS: COVID-19; Pandemics; Schizophrenia

\section{INTRODUÇÃO}

A 18 de março de 2020 foi declarado o primeiro estado de emergência devido à pandemia de COVID-19 em Portugal. Foi decretado o recolher obrigatório exceto para atividades essenciais durante 45 dias. Estima-se que cerca de $40 \%$ da população ativa tenha cumprido a indicação de recolher obrigatório. ${ }^{1}$

Alguns estudos procuraram compreender quais os fatores associados a maior adesão às medidas de saúde pública implementadas para controlar a pandemia confinamento domiciliário, distanciamento social, uso de máscara, desinfeção das mãos e superfícies. Foi encontrada uma relação positiva entre o cumprimento das medidas e fatores pessoais e sociais como a emocionalidade (na escala de personalidade HEXACO-60), a empatia, a confiança na ciência, o medo da doença, a capacidade de perceção das medidas impostas, a orientação política e o tipo de apoios económicos disponibilizados pelos governos. ${ }^{2}$

A pandemia de COVID-19 e as medidas para o seu controle podem ter causado ou acentuado sensações de ansiedade, solidão, desesperança, medo da morte ou ideação suicida. ${ }^{3-5}$ Estudos feitos em vários países (China, Itália, Espanha, Estados Unidos, Brasil, Portugal) mostraram aumento da ansiedade e depressão na população em geral, com valores que variaram entre os $23 \%$ e os 72\%. ${ }^{6}$ Um estudo feito em Portugal e no Brasil de maio a julho de 2020 em 555 pessoas detetou ansiedade significativa em 71\% dos indivíduos e depressão em 25\%. ${ }^{6}$ Outro estudo efetuado na população portuguesa em março de 2020, usando dados de mais de 11000 questionários aplicados online, revelou que 49,2\% das pessoas classificou o impacto psicológico da pandemia como moderado ou grave. ${ }^{7}$
Os efeitos da pandemia de COVID-19 na saúde mental divergem em diferentes grupos populacionais e podem ser mais acentuados em pessoas com doença mental. Um estudo de coorte efetuado na Holanda em 2748 pessoas com e sem doença mental (depressão, ansiedade e perturbação obsessivo-compulsiva) comparou respostas a questionários antes e depois do início da pandemia de COVID-19 e concluiu que os indivíduos com doenças mentais mais graves tiveram um maior impacto psicológico e mais dificuldade em usar estratégias adaptativas - mais problemas de sono, mais emoções negativas, pior alimentação, menos atividade física, mais alcoolismo, maior consumo de tabaco e incapacidade de manter redes sociais quando em isolamento. ${ }^{8}$

Não há muitos estudos publicados sobre o impacto da pandemia de COVID-19 em doentes com esquizofrenia. Um estudo efetuado na China em 60 doentes internados com esta patologia mostrou que a epidemia de COVID-19 causou um aumento dos níveis de ansiedade, depressão, problemas de sono e aumento dos marcadores inflamatórios. ${ }^{4}$

O objetivo do presente estudo é avaliar o impacto do confinamento devido à pandemia de COVID-19 numa amostra de doentes com esquizofrenia, em contexto de ambulatório.

\section{MÉTODOS}

O estudo observacional decorreu de julho a dezembro de 2020, na consulta externa do serviço de psiquiatria do Hospital de Vila Franca de Xira, tendo sido incluídos 40 doentes avaliados em consulta presencial pela primeira autora com diagnóstico de esquizofrenia ou outras psicoses não orgânicas (F20 a F29 segundo a 
CID-10). Foram incluídos todos os doentes maiores de 18 anos com esse diagnóstico que vieram a consultas presenciais e que aceitaram participar no estudo, tendo sido excluídos os doentes que já tinham estado infetados com SARS-CoV-2 e os doentes avaliados em consultas telefónicas.

Foram recolhidos dados demográficos e clínicos: sexo, idade, ocupação, estado civil, anos de duração da doença, toma regular de medicação antipsicótica injetável, toma regular de medicação ansiolítica ou hipnótica benzodiazepínica e acompanhamento em psicoterapia.

Foram colocadas as seguintes questões aos doentes pelo médico assistente, durante uma consulta de rotina:

- Sentiu que durante o confinamento pela epidemia de COVID-19 ficou mais ansioso que habitualmente?

- Passou a sair de casa com menos frequência que habitualmente?

- Deixou de tomar ou reduziu a sua medicação?

- Aumentou a dose da sua medicação ou associou outros medicamentos que não tomava habitualmente?

TABELA 1. Características clínicas e demográficas dos doentes.

\begin{tabular}{|l|c|}
\hline N=40 & \\
\hline Idade média & 40 anos \\
\hline Duração média da doença & 15 anos \\
\hline Sexo masculino & $60 \%$ \\
\hline Reformado/a por invalidez & $60 \%$ \\
\hline Solteiro/a & $75 \%$ \\
\hline Medicação antipsicótica injetável & $45 \%$ \\
\hline Medicação diária benzodiazepínica & $65 \%$ \\
\hline Psicoterapia regular & 0 \\
\hline Fumador/a & $52,5 \%$ \\
\hline Consome álcool diariamente & $17,5 \%$ \\
\hline Consome cannabis regularmente & $7,5 \%$ \\
\hline
\end{tabular}

TABELA 2. Percentagem de doentes que respondeu "sim" às questões.

\begin{tabular}{l|c} 
& \\
$\begin{array}{l}\text { Sente que o confinamento devido ao COVID-19 } \\
\text { o deixou mais ansioso que habitualmente }\end{array}$ & $37,5 \%$ \\
\hline $\begin{array}{l}\text { Passou a sair de casa com menos frequência que } \\
\text { habitualmente }\end{array}$ & $65 \%$ \\
\hline $\begin{array}{l}\text { Deixou de tomar ou reduziu a sua medicação } \\
\text { Aumentou a dose da sua medicação ou associou }\end{array}$ & $2,5 \%$ \\
\hline outros medicamentos & $15 \%$ \\
\hline $\begin{array}{l}\text { Aumentou o consumo de cannabis } \\
\text { Aumentou o consumo de tabaco }\end{array}$ & 0 \\
\hline Aumentou o consumo de álcool & $30,7 \%$ \\
\hline $\begin{array}{l}\text { Contactou o seu médico para antecipar consulta } \\
\text { ou veio à urgência de psiquiatria }\end{array}$ & $42,8 \%$ \\
\hline Sentiu necessidade de ter apoio psicológico & $10 \%$ \\
\hline
\end{tabular}

- Aumentou o consumo de cannabis/tabaco/álcool (se consumia anteriormente)?

- Contactou o seu médico para antecipar consulta ou veio à urgência de psiquiatria?

- Se não tinha acompanhamento psicológico prévio, sentiu necessidade de pedir apoio psicológico ou contactou a linha de apoio psicológico disponibilizada pelo SNS?

As informações clínicas e os resultados do questionário foram registados numa base de dados em Microsoft Excel e os dados foram analisados no programa estatístico JASP 0.13.1.0. Os doentes assinaram um consentimento informado autorizando a sua participação no estudo, que foi aprovado pela Comissão de Ética do Hospital Vila Franca de Xira.

\section{RESULTADOS}

As características demográficas e clínicas dos doentes estão registadas na Tabela 1.

As respostas às questões estão registadas na Tabela 2.

Na Tabela 3 comparam-se os doentes que responderam não ou sim à questão 1.

\section{DISCUSSÃO}

Os doentes com esquizofrenia e outras psicoses podem reagir à pandemia de forma diferente de doentes com outras perturbações mentais. Pode até haver um efeito positivo numa fase inicial do confinamento, ao reduzir a ansiedade social, normalizando a vivência de estar isolado em casa sem contactos sociais. ${ }^{5,8}$ Os efeitos negativos podem estar relacionados com diversos fatores. Os doentes com esquizofrenia podem ser menos propensos a pedir ajuda em situações de maior ansiedade e stress. ${ }^{9}$ Os défices cognitivos associados à esquizofrenia podem dificultar a adesão às normas de saúde pública, que são muitas vezes modificadas e adaptadas ao longo do tempo, exigindo flexibilidade cognitiva para as cumprir de forma ajustada. ${ }^{10,11}$ As suas redes de apoio social, que em alguns casos podem ser mais limitadas, podem ficar gravemente comprometidas durante o confinamento, causando rutura de relações afetivas, desemprego ou situação de sem-abrigo. ${ }^{5}$ A ansiedade e o isolamento podem aumentar os consumos de álcool e drogas, agravados pelo facto do acesso a consultas para problemas de adição estar comprometido. ${ }^{12,13} \mathrm{O}$ confinamento pode agravar hábitos de vida pouco saudáveis, como alimentação deficitária, falta de exercício físico, falta de exposição a luz solar. ${ }^{5}$ Pode haver dificuldades 
TABELA 3. Diferenças entre doentes que responderam "não" e "sim" à questão 1.

\begin{tabular}{|c|c|c|c|}
\hline & $\begin{array}{c}\text { Não se } \\
\text { sentiu mais ansioso } \\
(62,5 \%)\end{array}$ & $\begin{array}{c}\text { Sentiu-se } \\
\text { mais ansioso }(32,5 \%)\end{array}$ & $\begin{array}{l}\text { Diferença } \\
\text { estatisticamente } \\
\text { significativa }\end{array}$ \\
\hline Idade média & 43 & 41 & NS \\
\hline Duração média da doença & 15 & 16 & NS \\
\hline Sexo masculino & $64 \%$ & $53,3 \%$ & NS \\
\hline Reformado/a por invalidez & $60 \%$ & $60 \%$ & NS \\
\hline Solteiro/a & $76 \%$ & $73,3 \%$ & NS \\
\hline Medicação antipsicótica injetável & $32 \%$ & $66,7 \%$ & $\times 2-4.5, p-0.033$ \\
\hline Medicação diária com benzodiazepinas & $60 \%$ & $73,3 \%$ & NS \\
\hline Fumador/a & $52 \%$ & $53,3 \%$ & NS \\
\hline Consome álcool diariamente & $16 \%$ & $20 \%$ & NS \\
\hline Consome cannabis regularmente & $8 \%$ & $6,7 \%$ & NS \\
\hline Passou a sair de casa com menos frequência & $64 \%$ & $66 \%$ & NS \\
\hline Deixou de tomar ou reduziu a sua medicação & $4 \%$ & 0 & NS \\
\hline Aumentou a medicação & $8 \%$ & $26,6 \%$ & NS \\
\hline Aumentou o consumo de tabaco & $36,3 \%$ & $50 \%$ & NS \\
\hline Aumentou o consumo de álcool & 0 & $100 \%$ & $\times 2-7, p-0.008$ \\
\hline Antecipou consulta ou veio à urgência & $8 \%$ & $13 \%$ & NS \\
\hline Sentiu necessidade de ter apoio psicológico & $4 \%$ & $33,3 \%$ & $\times 2-6.3, p-0.012$ \\
\hline
\end{tabular}

no acesso a consultas de psiquiatria, psicologia, terapia ocupacional e enfermagem para administração de medicação injetável, com consequentes repercussões em termos de agravamento da sintomatologia e recaídas da doença. 3,10,11 As alucinações e delírios podem interferir com a avaliação por teleconsulta ou videochamada.

O risco de contrair coronavírus pode estar aumentado nos doentes com esquizofrenia: menor imunidade, maior presença de fatores de risco como obesidade e diabetes, limitações cognitivas que impedem a compreensão adequada de formas de prevenção de doença, dificuldades no acesso a cuidados de saúde por fatores socioeconómicos, menor qualidade de cuidados médicos por dificuldades na comunicação clara das queixas e pelo estigma. ${ }^{10-12,14}$

Nesta amostra de 40 doentes com esquizofrenia, cerca de um terço (32,5\%) sentiu maior ansiedade durante o confinamento devido à pandemia de COVID-19. Esta percentagem está de acordo com a obtida nos vários estudos disponíveis (valores entre os $23 \%$ e os $72 \%$ ), mas é ligeiramente inferior à obtida no estudo feito em março na população portuguesa (49,2\%) - as características demográficas desta amostra de doentes são, no entanto, muito diferentes dessa amostra populacional, que incluiu maioritariamente mulheres jovens e com formação universitária. ${ }^{7}$

O confinamento foi respeitado por cerca de dois terços dos doentes (65\%), o que é superior aos dados de que dispomos para a população em geral (40\%), e que demonstra aparentemente a boa capacidade de estes doentes cumprirem as indicações de saúde pública, ao contrário do que é sugerido na literatura consultada.

A grande maioria dos doentes (97,5\%) não parou ou reduziu a medicação e uma pequena percentagem de doentes (15\%) aumentou dose, possivelmente como consequência do aumento da ansiedade. Muitos doentes aumentaram os seus consumos de álcool (42,8\%) e tabaco (30,7\%), mas não houve aumento nos consumos de cannabis - o que poderá ser explicado pela menor facilidade em comprar produtos derivados da cannabis devido ao confinamento. $\bigcirc$ aumento da medicação e dos consumos de álcool e tabaco foi mais evidente em doentes que referiram estar ansiosos com o confinamento (no caso do álcool esta diferença foi estatisticamente significativa).

Houve um ligeiro aumento na procura de ajuda psiquiátrica ou psicológica (10\% e 15\% respetivamente), mas mais uma vez esses valores foram maiores em doentes que referiram estar ansiosos com o confinamento (no caso da necessidade de ajuda psicológica essa diferença foi estatisticamente significativa - 4\% vs 33,3\%).

Nenhuma variável clínica ou demográfica estudada se associou de forma estatisticamente significativa à maior ansiedade com o confinamento, exceto o uso de medicação injetável depot. Os doentes medicados com injetável de longa duração tiveram ansiedade em 66,7\% dos casos vs 32\% dos outros doentes. Uma possível justificação poderá ser o facto de os doentes que fazem medicação injetável terem contacto regular e necessariamente presencial com o centro de saúde ou serviço 
de psiquiatria do hospital, e nesses contactos ser feita sensibilização para os riscos da COVID-19, o que pode ter aumentado o seu nível de informação, mas também de preocupação com a pandemia. Outra justificação possível é o facto de doentes medicados com injetável terem geralmente patologia mais grave e, nessa medida, estarem mais propensos a ter ansiedade mais frequente e intensa.

\section{CONCLUSÃO}

A epidemia de COVID-19 e o confinamento usado para travar a sua propagação tiveram impacto neste grupo de doentes com esquizofrenia. Nalguns doentes houve aumento da ansiedade, dos consumos de álcool e tabaco, da dose da medicação e de recurso a consulta de psiquiatria urgente. Os doentes não relataram redução significativa da adesão à terapêutica. Verificou-se que a maioria dos doentes com esquizofrenia cumpriu as regras de confinamento impostas. No início de 2021 houve necessidade de novo confinamento, pelo que futuros estudos poderão avaliar melhor o impacto a longo prazo da pandemia e destes confinamentos nos doentes com esquizofrenia.

\section{RESPONSABILIDADES ÉTICAS}

CONFLITOS DE INTERESSE: Os autores declaram a inexistência de conflitos de interesse na realização do presente trabalho.

FONTES DE FINANCIAMENTO: Não existiram fontes externas de financiamento para a realização deste artigo.

CONFIDENCIALIDADE DOS DADOS: Os autores declaram ter seguido os protocolos da sua instituição acerca da publicação dos dados de doentes.

PROTEÇÃO DE PESSOAS E ANIMAIS: Os autores declaram que os procedimentos seguidos estavam de acordo com os regulamentos estabelecidos pelos responsáveis da Comissão de Investigação Clínica e Ética e de acordo com a Declaração de Helsínquia de 2013 da Associação Médica Mundial.

PROVENIÊNCIA E REVISÃO POR PARES: Não comissionado; revisão externa por pares.

\section{ETHICAL DISCLOSURES}

CONFLICTS OF INTEREST: The authors have no conflicts of interest to declare.

FINANCING SUPPORT: This work has not received any contribution, grant or scholarship
CONFIDENTIALITY OF DATA: The authors declare that they have followed the protocols of their work center on the publication of data from patients.

PROTECTION OF HUMAN AND ANIMAL SUBJECTS: The authors declare that the procedures were followed according to the regulations established by the Clinical Research and Ethics Committee and to the 2013 Helsinki Declaration of the World Medical Association.

PROVENANCE AND PEER REVIEW: Not commissioned; externally peer reviewed.

\section{REFERÊNCIAS}

1. PSE. Confinamento cai para 55\%. [acedido fevereiro 2021] Disponível em: https://www.pse.pt/confinamento-cai-para-55/.

2. Kuiper M, Bruijn A, Folmer C, Olthuis E, Brownlee E, Kooistra E, et al. The intelligent lockdown: compliance with COVID-19 mitigation measures in the Netherlands. Amsterdam Law School Research Paper No. 2020-20, General Subserie Research Paper No. 2020-02 [acedido fevereiro 2021] Disponível em: SSRN https://ssrn.com/abstract=3598215. doi: http:// dx.doi.org/10.2139/ssrn.3598215.

3. Amerio A, Andrea A, Odone A, Gianfredi V, Serafini G, Signorelli C, Amore M. COVID-19 pandemic impact on mental health of vulnerable populations. Acta Biomed. 2020;91:95-6. doi: 10.23750/abm.v91i9-S.10112.

4. Ma J, Hua T, Zeng K, Zhong B, Wang G, Liu X. Influence of social isolation caused by coronavirus disease 2019 (COVID-19) on the psychological characteristics of hospitalized schizophrenia patients: a case-control study. Transl Psych. 2020;10:411. doi: 10.1038/s41398-020-01098-5.

5. Brown E, Gray R, Monaco S, O'Donoghue B, Nelson B, Thompson A, et al. The potential impact of COVID-19 on psychosis: A rapid review of contemporary epidemic and pandemic research. Schiz Res. 2020;222:79-87.

6. Passos L, Prazeres F, Teixeira A, Martins C. Impact on mental health due to covid-19 pandemic: cross-sectional study in Portugal and Brazil. Int J Environ Res Public Health. 2020;17:6794. doi: 10.3390/ijerph17186794.

7. Paulino M, Dumas-Diniz R, Brissos S, Brites R, Alho L, Simões M, et al. COVID-19 in Portugal: exploring the immediate psychological impact on the general population. Psych Health Med. 2021;26:44-55. doi: 10.1080/13548506.2020.1808236.

8. Pan K, Kok A, Eikelenboom M, Horsfall M, Jörg F, Luteij RN, et al. The mental health impact of the COVID-19 pandemic on people with and without depressive, anxiety, or obsessive-compulsive disorders: a longitudinal study of three Dutch case-control cohorts. Lancet Psychiatry. 2020;8,2:121-9. doi: 10.1016/S2215-0366(20)30491-0.

9. Holzle P, Aly L, Frank W, Forstl H, Frank A. COVID-19 distresses the depressed while schizophrenic patients are unimpressed: A study on psychiatric inpatients. Psychiatry Res. 2020;291:113175. doi: 10.1016/j.psychres.2020.113175.

10. Padala SP, Dennis RA, Caceda R. Why COVID-19 Is Especially Difficult for Those With Schizophrenia: Reasons and Solutions. Prim Care Companion CNS Disord. 2020;22:20com02739. doi: 10.4088/PCC.20com02739.

11. Kozloff N, Mulsant B, Stergiopoulos V, Voineskos A. The covid-19 global pandemic: implications for people with schizophrenia and related disorders. Schiz Bull. 2020;46:752-7. doi: 10.1093/schbul/sbaa051. 
12. Fonseca L, Diniz E, Mendonca G, Malinowski F, Mari J, GadeIha A. Schizophrenia and COVID-19: risks and recommendations. Braz J Psychiatry. 2020;42:236-38. doi: 10.1590/15164446-2020-0010.

13. Ornella F, Moura H, Scherera J, Pechansky F, Kesslera F, Diemena L. The COVID-19 pandemic and its impact on substance use: Implications for prevention and treatment. Psychiatry Res. 2020;289:113096. doi: 10.1016/j.psychres.2020.113096.

14. Yao H, Chen J, Xu Y. Patients with mental health disorders in the COVID-19 epidemic. Lancet Psychiatry. 2020;7:e21. doi: 10.1016/S2215-0366(20)30090-0. 